\title{
EI ARS en el estudio y evaluación de metodología para el diseño de productos industriales: Aplicación y perspectiva
}

\author{
Dra. Deyanira Bedolla Pereda ${ }^{1}$, Dr. J orge Gil Tejeda \\ Departamento de Teoría y Procesos del Diseño, \\ División de Ciencias de la Comunicación y Diseño \\ Universidad Autónoma Metropolitana Unidad Cuajimalpa \\ Act. Alejandro Arnulfo Ruiz León \\ Departamento de Modelación Matemática de Sistemas Sociales \\ Laboratorio de Redes IIMAS \\ Universidad Nacional Autónoma de México
}

\section{Resumen}

Entre las tendencias actuales en el campo del diseño de productos industriales el Diseño sensorial (DS), presenta importantes implicaciones sociales, ya que responde a la reciente búsqueda de diseñadores e industrias por lograr la adecuación del objeto o sistemas de objetos industriales a las diferentes necesidades y preferencias de los consumidores - usuarios, con el objetivo de conducirlos a la presentación de cualidades funcionales y estéticas que respondan a las diferentes necesidades y preferencias de distintos grupos humanos, optimizando de este modo la interacción usuario - producto.

Las herramientas metodológicas que conforman el DS presentan actualmente un conjunto de necesidades de análisis, interpretación y estudio, ligadas a aspectos sociales del diseño de productos industriales, por lo cual se busca dar respuesta a estas necesidades de análisis mediante una herramienta que puede contribuir a su satisfacción: el análisis de redes sociales (ARS).

Palabras clave: productos industriales - implicaciones sociales - herramientas metodologicas - consumidores, usuarios.

\begin{abstract}
Among the current trends in the field of the product design manufacturers the sensory Design (DS), he presents important social implications, since it answers to the designers' recent search and industries for achieving the adequacy of the object or systems of industrial objects to the different needs and preferences of the consumers - users, with the aim to lead them to the presentation of functional and aesthetic qualities that answer to the different needs and preferences of different human groups, optimizing thus the interaction user - product.

The methodological tools that shape the DS present nowadays a set of needs of analysis, interpretation and study, tied to social aspects of the product design manufacturers, by which response is sought to give to these needs of analysis by means of a tool that it can contribute to his satisfaction: the analysis of social networks (ARS).
\end{abstract}

Key words: Industrial objects - Social implications - Methodological tools Consumers, users.

\footnotetext{
${ }^{1}$ Enviar correspondencia a: Deyanira Bedolla Pereda, dbedolla@correo.cua.uam.mx
} 
REDES- Revista hispana para el análisis de redes sociales

Vol.17,\#9, Diciembre 2009

http://revista-redes.rediris.es

\section{I ntroducción}

La competitividad empresarial que se ha venido incrementado enormemente en las ultimas décadas, obliga a industrias y diseñadores a encontrar nuevos métodos que permitan no solo optimizar y acortar el ciclo de diseño y desarrollo de los productos sino, incorporar al objeto el máximo valor en todos los ámbitos que lo caracterizan, asegurando de esta manera innovación, calidad y una mayor probabilidad de éxito y aceptación por el usuario.

De los cuatro ámbitos que conforman el esquema de configuración del diseño moderno (Rodríguez 2006): el funcional, tecnológico, comercial y expresivo (o estético), ha sido tradicionalmente el primero, el funcional, el que se había reconocido como crucial para el éxito del producto, sin embargo este concepto ha sufrido cambios considerables ya que en la actualidad se reconoce que el éxito de un objeto en el mercado puede ser determinado en gran medida por su apariencia estética y por el placer y la satisfacción que produce en el usuario (Khalid and Helander 2004, Helander and Khalid 2006). Así hoy se habla de la soft functionality (McDonagh-Philp and Lebbon 2000) para referirse a las necesidades emocionales y otros aspectos intangibles y cualitativos que afectan la relación del usuario con el producto.

Estudios teóricos del tema señalan la relevancia del aspecto emocional en la relación usuario- objeto; según la Theory of Retoric (Buchanan 1989) existen tres elementos interrelacionados que integran el aspecto comunicativo del diseño: technological reasoning, character y precisamente emotion, aspecto que según Buchanan es particularmente importante para la semántica del producto ya que puede derivarse tanto del contacto físico como de la contemplación del mismo.

De esta manera, es hoy búsqueda de numerosos diseñadores e industrias el lograr la adecuación del objeto o sistemas de objetos industriales a las diferentes inclinaciones y preferencias especificas de los diversos grupos de consumidores usuarios, de manera que sean capaces de presentar cualidades estéticas (o expresivas) que respondan a las diferentes necesidades y preferencias humanas, optimizando de este modo la interacción usuario - producto y aumentando sus probabilidades de aceptación en el mercado.

Actualmente esta búsqueda por adecuar el objeto al usuario, se traduce en la exploración de herramientas metodológicas que permitan tanto conocer como involucrar de manera central en el proceso de planeación y desarrollo del diseño de producto las preferencias y necesidades pertenecientes al ámbito psicoemocional del usuario o grupo de usuarios del objeto reflejadas en sus preferencias. 
REDES- Revista hispana para el análisis de redes sociales

Vol.17,\#9, Diciembre 2009

http: //revista-redes. rediris.es

Si bien tradicionalmente una disciplina como lo es el marketing ha buscado entender las preferencias, características y gustos del consumidor - usuario, sus tradicionales estrategias de determinación de preferencias y gustos a través de cuestionarios y otras averiguaciones de este tipo son un tanto superficiales y pragmáticas, útiles, para otros aspectos relacionados con el producto, pero no suficientes ni adecuados para su innovación (Vinyets 2000).

Así, dentro del ámbito del diseño existen diversidad de trabajos recientes que tienen como objetivo central llegar a identificar preferencias afectivas y las relaciones o percepciones emocionales de los usuarios acerca de los productos industriales. La mayoría de estos trabajos parten de la presentación de determinados objetos existentes a grupos de usuarios específicos para su evaluación, buscando llevar a cabo la identificación de aspectos como: dimensiones de valoración hedónica (Creusen, Snelders 2002), características de respuesta emocional (Desmet, Hekkert 2002), o bien el conjunto de cualidades estéticas percibidas (Jordan 2002), sin embargo conocer esta dimensión humana de esta manera se ha señalado como problemático ya que los usuarios la mayoría de las veces encuentran dificultades en expresar sus emociones y su sentir hacia los productos debido a que muchas veces no son necesariamente conscientes de ellos. (McDonagh et. al 2002)

Una de las herramientas que hemos propuesto recientemente para identificar e involucrar las características y necesidades de los diferentes grupos de usuarios holísticamente, es decir paralelamente características y necesidades físicas y psicoemocionales, es el diseño sensorial (DS) (Bedolla 2002), que a diferencia de las propuestas anteriormente señaladas no ha partido de la presentación de determinados objetos existentes a grupos de usuarios específicos, sino de la recopilación y análisis de numerosos estudios científicos pertenecientes al ámbito psicofisiológico y emocional humano. Esta perspectiva por su naturaleza busca dar a la temática un orden académico partiendo del análisis de la naturaleza multisensorial humana.

EI DS representa entonces un conjunto de herramientas metodológicas que complementan las actuales metodologías de diseño de productos industriales, herramientas que toman como elemento central para la identificación de características, necesidades e inclinaciones de grupos humanos diferenciados, una propiedad vital intrínseca a la naturaleza humana: los procesos sensoriales.

Los procesos sensoriales humanos, la percepción sensorial del individuo, representan una de las características humanas más trascendentes, que le permite 
REDES- Revista hispana para el análisis de redes sociales

Vol.17,\#9, Diciembre 2009

http://revista-redes.rediris.es

a toda persona estar en contacto constante con el mundo que le rodea. La sensorialidad es una cualidad de tal relevancia y amplitud que no solo abarca en todo individuo lógicamente dimensiones orgánicas, físicas, sino que paralelamente abarca dimensiones emocionales, por lo tanto define necesidades humanas de ámbito tanto físico como anímico ${ }^{2}$ a las que es posible satisfacer a través de los elementos que integran nuestro entorno artificial.

Los sentidos al ser las vías perceptivas de la información del entorno, tienen un papel vital en la aceptación, gusto o rechazo del producto ya que desde el nivel básico perceptivo un objeto, nos gustará o disgustará a través de la apreciación de la sensación en sí, de su cualidad e intensidad. En un nivel secundario continúa este proceso de evaluación del elemento a través de procesos más complejos emociones, sentimientos y deseos - procesos que consisten en excitación sensorial y valoración cognitiva a través de impresiones y recuerdos (Bedolla 2002).

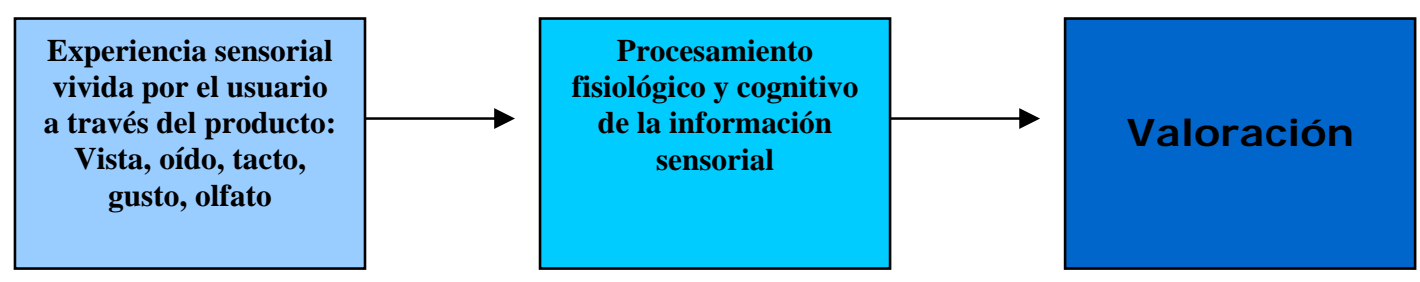

Figura 1. Secuencia de acontecimientos del origen de la valoración del producto por el usuario.

\section{Descripción de las herramientas metodológicas del DS}

El DS consiste en una serie de herramientas metodológicas destinadas a la innovación de objetos industriales mediante su diseño y rediseño. Esta serie de herramientas metodológicas las hemos denominado modelos guía los cuales:

- Identifican y presentan características, preferencias, e inclinaciones específicas de diferentes grupos de usuarios, derivadas directa e indirectamente de los procesos sensoriales humanos. Estos grupos de usuarios han sido caracterizados por las dos principales tendencias de personalidad (introvertidos y extrovertidos), los dos géneros humanos (femenino, masculino), y las diferentes etapas vitales del ser humano (infancia, adolescencia, edad adulta y adultos mayores)

- Identifican las cualidades tanto funcionales como estéticas o expresivas aplicables al producto industrial que satisfacen y/o responden a esas características, preferencias e inclinaciones humanas identificadas.

\footnotetext{
2 Las emociones son procesos dinámicos resultado de varias aportaciones cognitivas y biológicas de manera que pueden ser generadas tanto por el pensamiento como por los sentidos (Roussel y Woudzia 1986)
} 
REDES- Revista hispana para el análisis de redes sociales

Vol.17,\#9, Diciembre 2009

http://revista-redes.rediris.es

Ambos estudios han sido correlacionados y en base a estas correlaciones se han integrado una serie de modelos guía que constituyen herramientas metodológicas que complementando las actuales metodologías proyectuales, permiten la concepción y desarrollo de productos cuya forma de desempeñar sus funciones y sus características expresivas (forma, color, texturas, aromas, y sonidos que presente el objeto) responden a las capacidades, características, inclinaciones y preferencias especificas de diferentes grupos humanos, grupos de usuarios actuales o potenciales de productos industriales.

Las herramientas metodológicas fueron integradas de acuerdo a estudios científicos recopilados y analizados acerca de los siguientes aspectos principales:

- Análisis del proceso mediante el cual los sentidos humanos determinan el tipo y calidad de interacción de todo individuo con el producto, en base a la revisión de estudios científicos de áreas de la psicofisiología perceptiva humana principalmente.

- Identificación y estudio de grupos humanos diferenciados por sus inclinaciones, características y requerimientos físicos y psicoemocionales que condicionan y determinan la interacción con el objeto, en base a la revisión de estudios científicos de áreas de la fisiología y de la psicología humana.

- Identificación y análisis de las cualidades funcionales y estéticas del producto susceptibles de satisfacer necesidades, características e inclinaciones especificas de los diferentes grupos humanos identificados y estudiados, en base a trabajos pertenecientes a disciplinas como la estética experimental, la psicología del arte y el diseño industrial.

Así se realizó la integración de 9 modelos guía prácticos:

- 2 referentes a las dos principales tendencias de personalidad: introversión y extroversión esto es: se trata de modelos guía que describen las características, inclinaciones y preferencias de las personas que presentan una tendencia de personalidad principalmente hacia la extroversión o hacia la introversión, derivadas directa e indirectamente de los procesos sensoriales, y el conjunto de cualidades funcionales y expresivas que dan satisfacción a las mismas.

- 2 referentes a los dos géneros humanos: femenino y masculino que describen las características, inclinaciones y preferencias derivadas directa e indirectamente de los procesos sensoriales para cada género, y el conjunto de cualidades funcionales y expresivas que dan satisfacción a las mismas.

- 5 referentes a las diferentes etapas vitales humanas es decir modelos guía que describen las características, inclinaciones y preferencias derivadas directa e 
REDES- Revista hispana para el análisis de redes sociales

Vol.17,\#9, Diciembre 2009

http://revista-redes.rediris.es

indirectamente de los procesos sensoriales de la primera y segunda infancia, adolescencia, edad adulta y adultos mayores.

Todos estos modelos guía representan herramientas metodológicas que permiten al proyectista contar con unas guías que lo conducen a la innovación, especialización y personalización del producto de acuerdo a características inclinaciones y preferencias humanas especificas. La aplicación de los mismos durante el proceso de diseño del producto puede hacerse o bien aisladamente o paralelamente dos o tres de ellos ya que son complementarios.

A continuación se presenta como ejemplo uno de los modelos guía mencionados (Tabla 1 y Figura 2).

\begin{tabular}{|c|c|c|c|c|}
\hline & $\begin{array}{c}\text { Características } \\
\text { Sensoriales } \\
\text { Generales }\end{array}$ & $\begin{array}{l}\text { Particularidades } \\
\text { Sensoriales }\end{array}$ & $\begin{array}{c}\text { Atributos } \\
\text { Sensoriales } \\
\text { Involucrados }\end{array}$ & $\begin{array}{l}\text { Expresión } \\
\text { en el } \\
\text { Producto }\end{array}$ \\
\hline $\begin{array}{l}\text { Tendencias } \\
\text { Extrovertidas }\end{array}$ & $\begin{array}{l}\text { Tendencia a la } \\
\text { apetencia de estímulos } \\
\text { sensoriales fuertes. } \\
\text { Sistema nervioso fuerte, } \\
\text { umbrales altos. } \\
\text { Necesitan mas energía p } \\
\text { apreciar los estímulos } \\
\text { sensoriales. } \\
\text { Los extrovertidos } \\
\text { rendirán peor estando } \\
\text { relajados y mejor estand } \\
\text { activados o estresados } \\
\text { positivamente. } \\
\text { Los extrovertidos van a } \\
\text { ser buscadores de } \\
\text { sensaciones y } \\
\text { estímulos complejos, vari } \\
\text { y nuevos, que impacten a } \\
\text { sentidos. }\end{array}$ & $\begin{array}{l}\text { Mayor preferencia y } \\
\text { sensibilidad visual } \\
\text { para los colores que } \\
\text { para las formas. } \\
\text { Buscarán complacer } \\
\text { principalmente a la } \\
\text { vista, olfato y tacto. } \\
\text { Van a preferir figuras } \\
\text { complejas y } \\
\text { esquemáticas. } \\
\text { En su forma más } \\
\text { primitiva es amigo } \\
\text { del buen comer y de } \\
\text { tumbarse al sol; En } \\
\text { una forma más } \\
\text { refinada vive } \\
\text { alegremente } \\
\text { en una atmósfera } \\
\text { de amistades y } \\
\text { de arte. }\end{array}$ & $\begin{array}{l}\text { Visuales: Colores } \\
\text { principalmente, y } \\
\text { formas } \\
\text { Olores } \\
\text { Texturas }\end{array}$ & $\begin{array}{l}\text { Productos fuerter } \\
\text { caracterizados, } \\
\text { llamativos, } \\
\text { cambiantes variad } \\
\text { Amplio uso del } \\
\text { color. }\end{array}$ \\
\hline
\end{tabular}

Tabla 1. Requerimientos sensoriales de los individuos con tendencias de personalidad principalmente extrovertida y su expresión en el producto. 
Vol.17,\#9, Diciembre 2009

http: // revista-redes.rediris.es

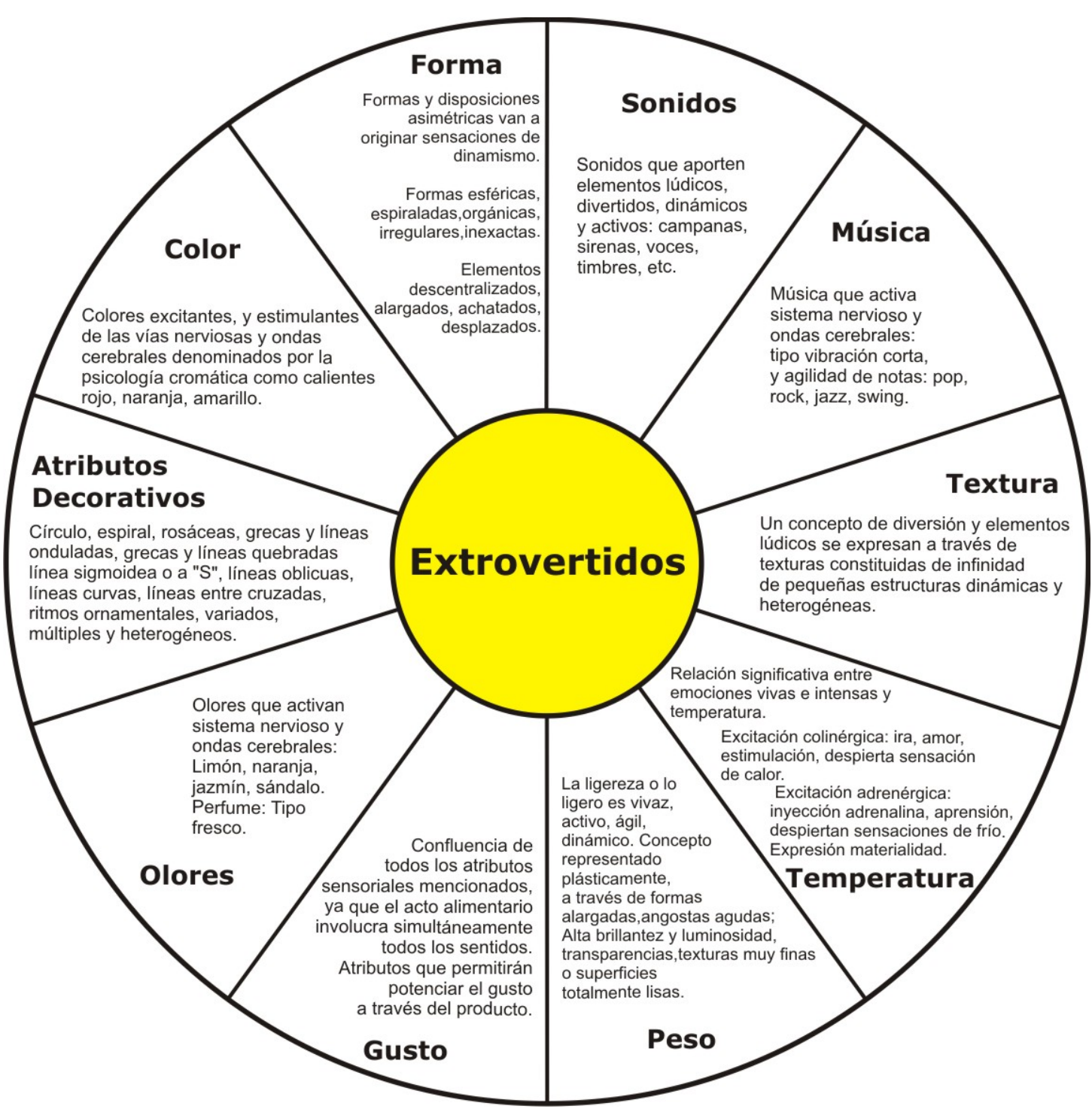

Figura 2. Atributos estéticos o expresivos que de acuerdo a sus propiedades y características están relacionados con la dimensión de personalidad extrovertido.

\section{Validación de las herramientas metodológicas mediante ARS}

Las herramientas metodológicas que conforman el DS presentan actualmente un conjunto de necesidades de análisis, interpretación y estudio, ligadas a aspectos sociales del diseño de productos industriales, por lo cual se busca dar respuesta a estas necesidades de análisis mediante una herramienta que puede contribuir a su satisfacción: el análisis de redes sociales (ARS).

Buscando traducir los diferentes elementos a tomar en cuenta en esta validación, es posible mencionar que en base a que una red social es un conjunto bien definido de actores- individuos, grupos, organizaciones, comunidades, sociedades globales, etc. - podemos identificar a los actores en el análisis del diseño sensorial (DS) de productos a los grupos de individuos con características específicas que evaluaron 
REDES- Revista hispana para el análisis de redes sociales

Vol.17,\#9, Diciembre 2009

http://revista-redes.rediris.es

los objetos rediseñados en base a las herramientas metodológicas preconcebidas, es decir los modelos guía.

En el DS podemos señalar a las relaciones sociales, como coincidencias, y/o diferencias en las preferencias sensoriales reflejadas en los productos, que indican el modelo sensorial adoptado por estos determinados sectores de la sociedad, sus aspiraciones y preocupaciones, sus divisiones, jerarquías e interrelaciones, mismas que enmarcan normas socialmente prescritas que condicionan la experiencia multisensorial perceptiva.

La identificación de estos aspectos son muy relevantes para la validación de los modelos guía propuestos ya que de acuerdo con Mitchell (1969) las "características de estos lazos como totalidad pueden ser usados para interpretar los comportamientos sociales de las personas implicadas".

Como primer paso para la validación de dichas herramientas metodológicas recientemente hemos desarrollado de manera experimental en base al DS, específicamente en base a tres de los modelos guía: el del género femenino, personalidad extrovertida y edad adulta, el rediseño de un conjunto de productos, específicamente de mouses o ratones (hardware), mismos que han sido sometidos a distintas evaluaciones por 55 usuarios, caracterizados por ser de género femenino, presentar tendencia de personalidad hacia la introversión y extroversión y del rango de edad que va de 18 a 45 años; las usuarias fueron elegidas al azar entre el profesorado, el personal administrativo y el alumnado de la Universidad Tecnológica de la Mixteca en Oaxaca México.

Las evaluaciones consistieron en:

- Preguntar el nivel de preferencia de las usuarias por el producto rediseñado (mouses) en base a los tres modelos guía, frente a otros productos similares, mediante su contemplación o percepción visual; de este modo, se presentaron contemporáneamente a las usuarias otros mouses pero con atributos plástico sensoriales cuya aplicación no fue en base a las herramientas del diseño sensorial (modelos guía).

- Conocer él o los diferentes atributos sensoriales, motivo por él o los que las usuarias eligieron uno de los mouses como el preferente, a través de su contemplación o percepción visual.

- Mediante la percepción táctil, manipulación y uso del producto, se les preguntó a las usuarias el nivel de preferencia del mouse rediseñado en base a los modelos 
REDES- Revista hispana para el análisis de redes sociales

Vol.17,\#9, Diciembre 2009

http://revista-redes.rediris.es

guía, frente a los otros mouses con atributos plástico sensoriales cuya aplicación no fue en base a las herramientas del diseño sensorial (modelos guía).

El procedimiento del proceso de evaluación fue el siguiente:

Se pidió a las personas si querían participar en el estudio voluntariamente.

Se les invitó a pasar a la habitación donde estaba el material de apoyo (los mouses junto a una computadora PC y teclado) para controlar la luz y la hora de la prueba.

Se les explicó lo que se les iba a presentar y lo que tendrán que hacer controlando el tiempo de respuesta.

Se anotaron en los cuestionarios creados especialmente para la prueba las respuestas dadas a las evaluaciones requeridas del producto.

Para el análisis y la interpretación de resultados se aplicó de manera experimental el análisis de redes sociales a los resultados, específicamente el software Pajek.

A continuación se muestra uno de los modelos matemáticos (red) surgido en esta aplicación experimental (Figura 3).

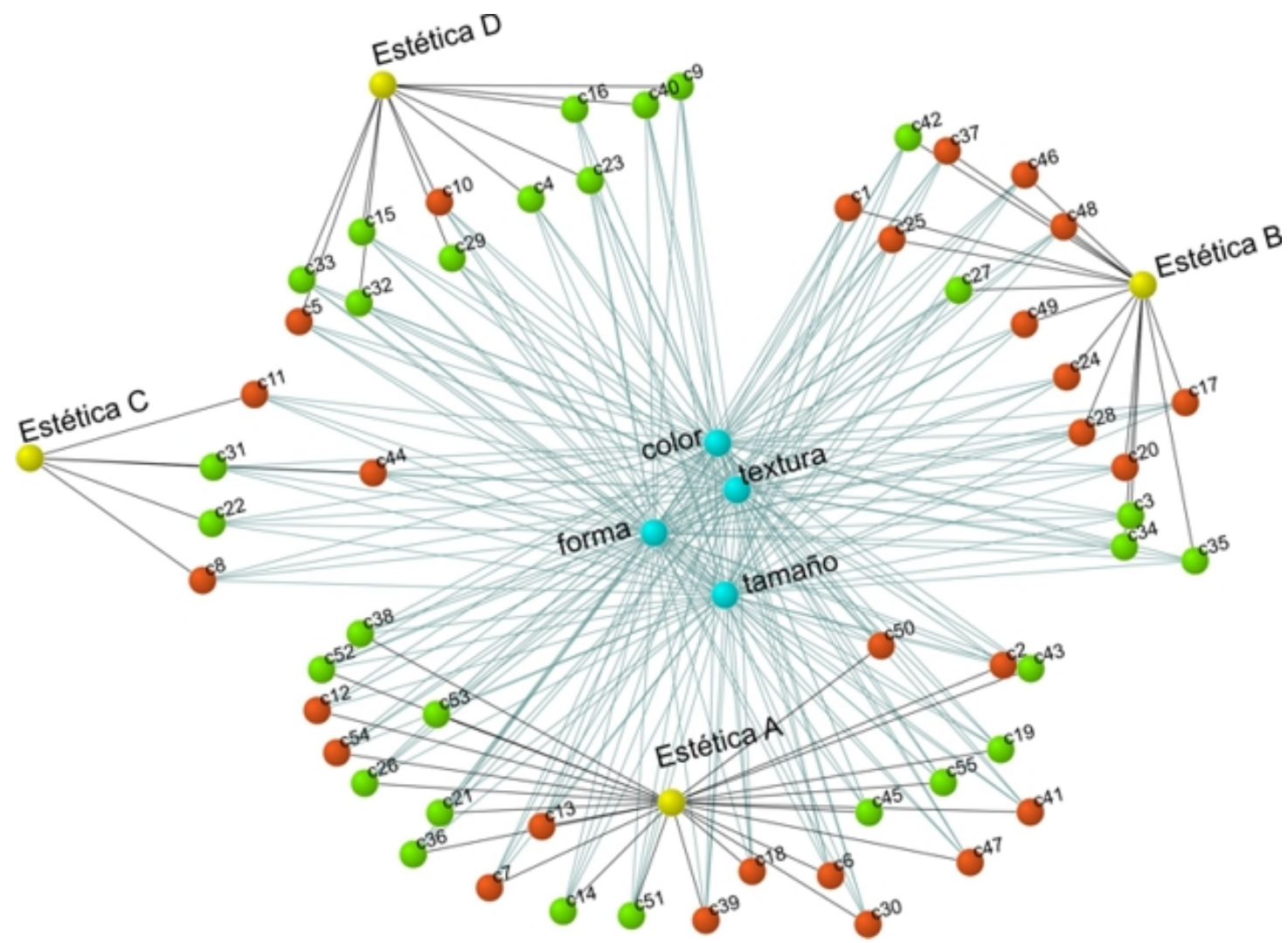

Figura 3. Red o modelo matemático que muestra las preferencias de 55 usuarias acerca de 4 rediseños multisensoriales de mouses o ratones (señalados con el color amarillo y el texto estética A, B, C, o D).

Las usuarias han sido caracterizadas primordialmente por su tendencia principal de personalidad: introvertidas (color verde), extrovertidas (color naranja). Al centro puede observarse el grado o nivel en que cada atributo sensorial que integraron los diferentes mouses generó la preferencia. 
REDES- Revista hispana para el análisis de redes sociales

Vol.17,\#9, Diciembre 2009

http://revista-redes.rediris.es

La red se compone de 63 nodos, 55 en forma circular que representan a los voluntarios en dos categorías, introvertidos en color verde y extrovertidos en color naranja, los 4 círculos amarillos representan a los mouses rediseñados en base a los modelos guía y los 4 círculos azules ubicados al centro de la red representan las cualidades de preferencia. La calificación que otorgó cada uno de los voluntarios a cada una de las cualidades de preferencia se tomó como el valor del vínculo entre éstos y cada una de ellas. También se consideró un vínculo de valor uno entre los voluntarios y el mouse que prefirió cada uno de ellos.

Al considerar el valor de los vínculos entre los voluntarios y las cualidades como una medida de disimilaridad, Pajek nos muestra el grado de influencia de cada una de las cualidades en la preferencia, según su posición en la red. El sistema toma en cuenta el valor de todos los vínculos para determinar la posición de cada uno de los nodos.

Aquellas cualidades a las que hayan otorgado la misma calificación todos los voluntarios se localizan en la parte central de la red, como es el caso de la forma que también resulta la de mayor influencia en la preferencia; en el caso del tamaño vemos que se localiza mas cerca de los nodos que representan a los voluntarios que prefirieron el mouse $A$, que de los que prefirieron a los otros mouses, lo que indica que es la segunda cualidad que se tomó en cuenta para su preferencia, así mismo vemos que el color y la textura son más importantes que el tamaño para aquellos que prefirieron al mouse $B$.

Los resultados en referencia específica a cada perfil de usuario mostraron:

a) Nivel de preferencia de las usuarias por el mouse rediseñado en base a las herramientas del DS mediante su contemplación o percepción visual:

Se comprobó que de 27 usuarias con tendencias extrovertidas (representadas en color naranja), 12 de ellas prefirieron en primer lugar el mouse rediseñado en base a la herramientas del DS (identificado como estética A), 10 de ellas como primera opción el mouse tradicional con cambios arbitrarios de color (azul) y de forma (muesca en uno de los extremos inferiores) (identificado como estética B), 3 de ellas como primera opción un mouse tradicional pero con otro tipo de cambios arbitrarios de color (naranja), y de forma (ligera forma orgánica mediante curvas) (identificado como estética C), 2 de ellas como primera opción el mouse tradicional sin cambio alguno (identificado como estética D). 
REDES- Revista hispana para el análisis de redes sociales

Vol.17,\#9, Diciembre 2009

http://revista-redes.rediris.es

De 28 usuarias con tendencias introvertidas 12 de ellas prefirieron en primer lugar el mouse rediseñado en base a la herramientas del DS (identificado como estética A), 9 de ellas como primera opción el mouse tradicional sin cambio alguno (identificado como estética D), 5 de ellas como primera opción el mouse tradicional con cambios arbitrarios de color (azul) y de forma (identificado como estética B), y 2 de ellas como primera opción un mouse tradicional pero con otro tipo de cambios arbitrarios de color (naranja), y de forma (ligera forma orgánica mediante curvas) (identificado como estética $\mathrm{C}$ ).

b) Nivel de preferencia del mouse rediseñado en base a los modelos guía, mediante su percepción táctil, es decir manipulación y uso del producto.

Se comprobó que de 27 usuarias con tendencias extrovertidas 17 de ellas eligieron como primera opción de preferencia el mouse tradicional con cambios arbitrarios de color (azul) y de forma (muesca en uno de los extremos inferiores) (identificado como estética B), 9 de ellas como primera opción el mouse rediseñado en base a la herramientas del DS (identificado como estética A), y una de ellas como primera opción el mouse tradicional pero con otro tipo de cambios arbitrarios de color (naranja), y de forma (ligera forma orgánica mediante curvas) (identificado como estética C).

De 28 usuarias con tendencias introvertidas 18 prefirieron en primer lugar el mouse rediseñado en base a la herramientas del DS (identificado con la letra A), 5 de ellas como primera opción el mouse tradicional sin cambio alguno (identificado como estética D), 3 de ellas como primera opción un mouse tradicional pero con otro tipo de cambios arbitrarios de color (naranja), y de forma (ligera forma orgánica mediante curvas) (identificado como estética C), y 2 de ellas como primera opción el mouse tradicional con cambios arbitrarios de color (azul) y de forma (muesca en uno de los extremos inferiores) (identificado como estética B)

En conclusión puede decirse que mediante la manipulación y uso del producto por los dos grupos de usuarias, el orden que se prefiguró de las preferencias cambió.

En el caso del grupo de usuarias extrovertidas, la preferencia que estuvo en el primer lugar, fue el mouse tradicional con dos ligeros cambios (de color y forma identificada como estética B). Las causas se explican debido a la variable sociocultural que no se ha tenido oportunidad de medir. Esta conclusión preliminar se realiza a partir de las afirmaciones que durante la evaluación de los productos, diversas usuarias de perfil extrovertido llevaron a cabo, como por ejemplo: "me acomoda", "ya me acostumbré", "parece mas tecnológico", "tiene accesorios", "tiene calidad, es de calidad". 
REDES- Revista hispana para el análisis de redes sociales

Vol.17,\#9, Diciembre 2009

http://revista-redes.rediris.es

La hipótesis que se deduce de lo anterior es que las usuarias prefieren usar ciertos productos tomando en cuenta aspectos ligados al valor de marca. Medir estas y otras variables es el objetivo fundamental que tenemos para continuar con el estudio y desarrollo de estas herramientas metodológicas para la innovación de objetos, de modo que sea posible llegar a concebir un instrumento que le permita a las diferentes industrias de nuestro país generar nuevos productos de mayor calidad desde la perspectiva multisensorial que traigan beneficios económicos al poder competir mas eficazmente.

\section{Perspectiva de desarrollo y aplicación del ARS en el diseño sensorial}

Como ha sido expuesto anteriormente, los modelos guía (herramientas metodológicas) que integran al DS, fueron integrados en base al estudio de dimensiones humanas que hemos denominado interno personales, es decir: las dos tendencias principales de personalidad (introvertido - extrovertido), los géneros humanos (femenino - masculino), y las edades o etapas vitales (primera y segunda infancia, adolescencia, edad adulta y adultos mayores).

En base a las conclusiones del estudio que hemos descrito a los largo de este trabajo, la continuación de este estudio será la búsqueda por validar experimentalmente estas herramientas metodológicas, pero considerando esta vez el análisis de diversas dimensiones que hemos denominado de "ámbito externo al sujeto", el objetivo será concretamente conocer cómo aspectos, como los son el nivel educativo, la ocupación y el origen geográfico determinan necesidades, características e inclinaciones sensoriales de los grupos humanos.

Existen escasos antecedentes de estudios que analizan la influencia de aspectos socioculturales en las preferencias, ya que puede decirse que en general ha habido poco interés en el estudio de los factores socioculturales que influyen en las preferencias estéticas; preferencias que han sido estudiadas en relación a la obra de arte, específicamente a la inclinación por ciertas tipologías de obras pictóricas, se trata de estudios que han tomado como elemento determinante del gusto estético a los factores socioculturales.

Bulley (1933) Ilama la atención de los psicólogos sobre la importancia de los factores socioculturales en la determinación de las preferencias estéticas. Así, considerando un amplio material: Diez mil juicios de adultos clasificados en categorías socioprofesionales o en niveles de formación, establece la existencia de una significativa conexión entre ciertas variables socioculturales y la seguridad del juicio estético. Tras comparar los resultados de juicios preferenciales, sobre pares 
REDES- Revista hispana para el análisis de redes sociales

Vol.17,\#9, Diciembre 2009

http://revista-redes.rediris.es

de cuadros de desigual valor, la autora descubre diferencias significativas entre la gente con estudios superiores y la que no los posee, y finalmente entre distintas profesiones.

Un interesante estudio más, es el que llevaron a cabo Knapp y Wulff (1963) los cuales realizaron una investigación relativa a las preferencias en materia de pintura figurativa o abstracta, estudiaron la relación entre el nivel de formación de los padres y las elecciones estéticas de niños de primer año, los sujetos se repartieron en tres grupos que se correspondían con el grado de formación de sus padres: Superior, media y básica: El análisis de variabilidad muestra la influencia del nivel escolar de los padres.

Marcus, Oprea, y Sandulescu (1972) por su parte toman la fidelidad de representación como criterio para medir el efecto de las variables sociales. Haciendo juzgar a adolescentes procedentes de clases sociales distintas cuadros con un cierto grado de realismo, así constataron que los adolescentes de medios sociales favorecidos tienden a valorar obras en las que la expresión personal del artista es preponderante.

El estudio que puede considerarse como el más sistemático entre los que buscan los determinantes socioculturales del gusto en materia de pintura, es la investigación llevada a cabo por Bernard (1972), en la que observó y analizó las compras efectuadas por 480 sujetos en una tienda de reproducciones de cuadros, el autor establece una correspondencia entre el contenido de las elecciones y el conjunto de las variables sociales o culturales que pueden caracterizar a cada uno de los sujetos.

Un análisis factorial permitió deducir una tipología de compradores aficionados compuesta de gente joven entre 20 y 30 años con un nivel superior de formación y pertenecientes a un medio en el que el proceso de aculturación es ya antiguo, los cuales mostraron una gran preferencia por la pintura moderna tanto de pintores abstractos como de cubistas, a los fauves, nabis, y de manera general a todos los post - impresionistas, algunos pintores como El Greco y Durero, se encuentran también entre las preferencias de este joven grupo.

Un segundo grupo de más edad con un grado de enseñanza superior o secundaria demostraron sentirse atraídos por los pintores antiguos de reconocido prestigio: Brueghel, Vermeer, toda la pintura francesa del siglo XVIII, los impresionistas y algunos pintores del siglo $X X$, en particular Vlaminck y Utrillo. El tercer grupo estaba formado por los más veteranos del muestreo, con 50 años o más con estudios de enseñanza media, demostraron una decidida oposición hacia la pintura 
REDES- Revista hispana para el análisis de redes sociales

Vol.17,\#9, Diciembre 2009

http://revista-redes.rediris.es

moderna, sus elecciones aparecieron relativamente eclécticas, guiadas únicamente por lo cuidado del tema tratado, demostraron ser grandes aficionados a los paisajes y a las flores, gustan en particular de los pintores holandeses, los paisajistas franceses del siglo XIX y algunos impresionistas como Ruysdael, Van de Velde, Corot y Sisley son sus favoritos. Entre las conclusiones Bernard destaca el efecto prioritario de la educación recibida.

En general las herramientas de análisis utilizadas en estos estudios son las pertenecientes al ámbito del análisis estadístico. La aplicación y aprovechamiento de las herramientas del análisis de redes sociales (ARS) en nuestro caso, nos permitirá un maneja adecuado de la información recogida a través de las pruebas con usuarios, pero sobre todo, la visualización de relaciones entre los diferentes elementos involucrados y su consiguiente análisis sistemático con bases matemáticas, permitiendo verificar o refutar nuestra hipótesis acerca de las causas de las preferencias en relación a productos industriales.

La antropóloga Constance Classen (1993) que se ha dedicado ampliamente al estudio de una antropología de los sentidos, ha señalado muy claramente la importancia y aportaciones de los estudios que buscan la identificación de las preferencias relacionadas con la percepción humana a través de algún o todo el conjunto de los sentidos "la construcción cultural de la percepción sensorial condiciona de modo fundamental nuestra experiencia y comprensión de nuestros cuerpos y del mundo. El modelo sensorial adoptado por una sociedad revela sus aspiraciones y preocupaciones, sus divisiones, jerarquías e interrelaciones. Por tanto, si los sentidos pueden compararse a ventanas... (éstos) enmarcan la experiencia perceptiva según normas socialmente prescritas".

Con la realización de estos estudios, pretendemos, innovar en el ámbito del diseño de productos mediante la aplicación de una nueva herramienta de análisis de resultados de las evaluaciones del producto industrial por parte de grupos de usuarios: el análisis desde el enfoque de las redes sociales; igualmente consideramos que es posible innovar en el ámbito de la teoría de las redes sociales, mediante la aplicación de las herramientas que permiten el análisis de redes sociales en una nueva área del conocimiento en la que hasta ahora no se había empleado: la concepción y desarrollo de productos industriales. 
REDES- Revista hispana para el análisis de redes sociales

Vol.17,\#9, Diciembre 2009

http: //revista-redes. rediris.es

\section{Bibliografia}

Bedolla Pereda Deyanira (2002). Diseño Sensorial la Nuevas Pautas para la Innovación Especialización y Personalización del Producto (tesis doctoral), Base de datos para tesis doctorales de la Universidad Politécnica de Cataluña (UPC): www.tdx.cesca.es, Barcelona, ISBN: 84-688-0748-6; Depósito Legal: B-4730-2003.

Bernard Y. (1972) "Sex ilnfluence in aesthetic behavior". Perceptual and motor skills, 34

Buchanan R. (1989). Declaration by design: Rethoric, argument, and demonstration in design practice. In: Design discourse: History, Theory, Criticism. Edited by: Margolin, V. University of Chicago Press. Chicago.

Bulley M. (1933). Have you good taste? Methuen, Londres

Classen Constance (1993), Worlds of sense: Exploring the senses in history and across cultures. Londres: Routledge.

Creusen M., Snelders Dirk (2002). Product appearance and consumer pleasure. In Pleasure with products. Edited by: Green William S., and Jordan Patrick W., (Eds), (Taylor \& Francis), London and New York.

Desmet P. M., Hekkert P.P. (2002). The basis of product emotions. In Pleasure with products. Edited by: Green William S., and Jordan Patrick W., Taylor \& Francis, London and New York.

Helander M. G., Khalid H. M. (2006). Affective and pleasurable design. In: Handbook on Human Factors and Ergonomics, edited by Salvendy, G., Wiley, New York.

Jordan Patrick W. (2002). The personalities of products. In: Pleasure with products, Edited by: Green William S., and Jordan Patrick W., (Taylor \& Francis. London and New York).

Khalid H. M., Helander M. G. (2004). "A framework for affective customer needs in product design". Theor. Issues Ergon. Sci.,5 (1).

Knapp, R.H., Wulff A. (1963). "Preferences for abstract and representational art". J ournal of Social Psychology, 60

Marcus S., Oprea P. y Sandulescu M. (1972). "Psychosocial factors in the reaction to certain modern paintings ». Revue roumaine des sciences sociales: Sèrie de psychologie, 16

McDonagh D., Bruseberg A., Haslam Ch. (2002). "Visual product evaluation. Exploring users' emotional relationships with products". Applied Ergonomics, 33.

McDonagh-Philp D., and Lebbon C., (2000) "The emotional domain in product design". Des. J., 3 (1).

Mitchell J.C., (1969), Social Networks in Urban Settings. Manchester. England: Manchester University Press.

Rodríguez Morales, Luis (2006). Diseño, estrategia y táctica, Diseño y Comunicación. Siglo XXI editores, México.

Rusell J. A.., and Woudzia L. (1986). "Affective judgments, common sense, and Zajonc's thesis of independence". Motivation and emotion, 10.

Vinyets, Joan (2002). "Per un altro marketing, il nuovo design si basa sull'esperienza". Domus 826, Mayo. 